\title{
Long-Term Plasticity in Mouse Sensorimotor Circuits after Rhythmic Whisker Stimulation
}

\author{
Pierre Mégevand, ${ }^{1,2 \star}$ Edgardo Troncoso, ${ }^{1 \star}$ Charles Quairiaux, ${ }^{1 \star}$ Dominique Muller, ${ }^{1}$ Christoph M. Michel, ${ }^{1,2}$ and \\ Jozsef Z. Kiss ${ }^{1}$ \\ ${ }^{1}$ Department of Fundamental Neuroscience, Faculty of Medicine, University of Geneva, 1211 Geneva 4, Switzerland, and ${ }^{2}$ Functional Brain Mapping \\ Laboratory, Department of Clinical Neuroscience, Geneva University Hospitals and University of Geneva, 1211 Geneva 4, Switzerland
}

\begin{abstract}
Mice actively explore their environment by rhythmically sweeping their whiskers. As a consequence, neuronal activity in somatosensory pathways is modulated by the frequency of whisker movement. The potential role of rhythmic neuronal activity for the integration and consolidation of sensory signals, however, remains unexplored. Here, we show that a brief period of rhythmic whisker stimulation in anesthetized mice resulted in a frequency-specific long-lasting increase in the amplitude of somatosensory-evoked potentials in the contralateral primary somatosensory (barrel) cortex. Mapping of evoked potentials and intracortical recordings revealed that, in addition to potentiation in layers IV and II/III of the barrel cortex, rhythmic whisker stimulation induced a decrease of somatosensory-evoked responses in the supragranular layers of the motor cortex. To assess whether rhythmic sensory input-based plasticity might arise in natural settings, we exposed mice to environmental enrichment. We found that it resulted in somatosensory-evoked responses of increased amplitude, highlighting the influence of previous sensory experience in shaping sensory responses. Importantly, environmental enrichment-induced plasticity occluded further potentiation by rhythmic stimulation, indicating that both phenomena share common mechanisms. Overall, our results suggest that natural, rhythmic patterns of whisker activity can modify the cerebral processing of sensory information, providing a possible mechanism for learning during sensory perception.
\end{abstract}

\section{Introduction}

The acquisition of sensory information is an active process where sensory input and motor behavior influence each other. When exploring their environment, mice and rats actively move their facial vibrissae over objects and surfaces in rhythmical sweeps at frequencies ranging between 5 and $15 \mathrm{~Hz}$ (Carvell and Simons, 1990; Wolfe et al., 2008). This form of active sensation has been compared with digital palpation and microsaccadic eye movements in primates (Carvell and Simons, 1990; Kleinfeld et al., 2002). A consequence of the rhythmical nature of whisking is that neuronal activity in the primary somatosensory (barrel) cortex varies rhythmically in synchrony with whisker movement and contact against obstacles (Fee et al., 1997; Crochet and Petersen, 2006; von Heimendahl et al., 2007). The vibrissal motor cortex, which controls whisker movement (Brecht et al., 2004), is interconnected with both cortical and subcortical somatosensory structures (Miyashita et al., 1994), and activity in the motor cor-

\footnotetext{
Received Dec. 16, 2008; revised March 3, 2009; accepted March 25, 2009.

This work was supported by Swiss Academy of Medical Sciences Grant 323600-111505 (M.D.-Ph.D. Program of the Swiss Universities) to P.M., by Swiss National Science Foundation Grant 320000-111783 to C.M.M., and by Swiss National Foundation Grant 3100A0-116613/1, the Eagle Foundation, and the European Community Grant Promemoria No. 512012-2005 to J.Z.K. We thank Katalin Korodi for helpful discussions and Cynthia Saadi for technical assistance with the histological preparations. The Cartool software (http://brainmapping.unige.ch/Cartool.php) is developed by Denis Brunet, from the Functional Brain Mapping Laboratory, Geneva, supported by the Center for Biomedical Imaging, Geneva and Lausanne, Switzerland.

*P.M., E.T., and C.Q. contributed equally to this work.

Correspondence should be addressed to JozsefZ. Kiss at the above address. E-mail: jozsef.kiss@medecine.unige.ch.

D01:10.1523/JNEUROSC1.5965-08.2009

Copyright $\odot 2009$ Society for Neuroscience $\quad 0270-6474 / 09 / 295326-10 \$ 15.00 / 0$
}

tex is also modulated synchronously with whisker movement (Kleinfeld et al., 2002; Chakrabarti et al., 2008).

How exactly this rhythmic whisking activity can affect the processing of sensory information in the somatosensory-motor cortical network remains, however, essentially unknown. Synchronous rhythmical activity in and across cortical areas is thought to play a key role in facilitating neuronal communication and in establishing functional links between neuronal populations (Varela et al., 2001; Erchova and Diamond, 2004; Fries, 2005). It might also be that this activity facilitates the generation of long-term adaptive changes in cortical function that underlie perception (Gilbert et al., 2001). Recent evidence suggests that periods of rhythmical visual or auditory stimulation can induce properties of synaptic plasticity such as long-term potentiation of cortical responses evoked by sensory inputs (Clapp et al., 2005, 2006; Teyler et al., 2005). Whether similar mechanisms could be related to whisking activity and whether such changes could be behaviorally relevant is not known. To address these issues, we used multichannel epicranial recordings of somatosensoryevoked potentials and laminar intracortical recordings (Troncoso et al., 2000, 2004; Mégevand et al., 2008) to test in anesthetized mice whether a period of passive rhythmical whisker stimulation at $8 \mathrm{~Hz}$, the dominant frequency during exploratory whisking behavior (Carvell and Simons, 1990), could induce long-lasting changes of evoked sensory responses in the barrel and motor cortices. Furthermore, to explore the behavioral relevance of these effects, we compared them with changes possibly evoked by exposure of the animals to environmental enrichment. Overall, our results suggest that cortical plasticity induced by 
rhythmic sensory input is a relevant mechanism for the adjustment of cortical activity during active sensory perception.

\section{Materials and Methods}

Animals. Male C57BL/6 mice aged 3-6 months were used for the experiments. All procedures were in accordance with Swiss laws and were approved by the Ethics Committee on Animal Experimentation of Geneva University Medical School and by the Veterinary Office of Geneva.

Electrophysiological recordings. As detailed previously (Troncoso et al., 2000, 2004; Mégevand et al., 2008), a custom-designed electromechanical device was used for stimulating whiskers. Stimuli consisted of $300 \mu \mathrm{m}$ back-and-forth deflections with $1 \mathrm{~ms}$ rise time, applied to all whiskers on one side of the snout, $1 \mathrm{~cm}$ away from the face [30-100 stimuli per somatosensory-evoked potential (SEP), 2-3 s interstimulus interval]. Mice were anesthetized with intraperitoneal pentobarbital $(60 \mathrm{mg} / \mathrm{kg}$ complemented by $20 \mathrm{mg} / \mathrm{kg}$ each hour as needed) during the epicranial single-channel SEP recordings; during the longer epicranial SEP mapping and intracortical recordings, inhaled isoflurane $(0.8-1 \%$ in $20 \%$ oxygen $/ 80 \%$ air) was used to maintain stable anesthesia over a longer time period, as requested by the Veterinary Office of Geneva. Mice were placed in a stereotaxic frame, the scalp was anesthetized with bupivacaine, and the skull bones were widely exposed. Anesthetic depth was monitored clinically and kept constant so as to almost completely suppress the hindlimb withdrawal reflex. Body temperature was maintained at $37^{\circ} \mathrm{C}$ by a feedback-controlled heating blanket (Harvard Apparatus). During epicranial single-channel SEP recordings, an array of five stainless steel macro-electrodes (500 $\mu \mathrm{m}$ diameter) was dipped in EEG paste (EC2; Grass Technologies) and applied onto the parietal bone overlying the barrel cortex. Recordings were referenced to a frontal midline electrode. Signals were amplified, filtered ( $4 \mathrm{~Hz}$ high-pass, $300 \mathrm{~Hz}$ low-pass), digitized $(2000 \mathrm{~Hz}$ sampling rate, 16-bit resolution; S. Czellar, CERN, Geneva, Switzerland), and displayed on screen and stored on hard drive for subsequent analysis. During epicranial SEP mapping recordings, an array of 32 equidistant stainless steel electrodes (500 $\mu \mathrm{m}$ diameter) was dipped in EEG paste and applied onto the frontal and parietal bones of both hemispheres. A midline parietal electrode was used as the reference. Signals were amplified, filtered (1 Hz high-pass, $500 \mathrm{~Hz}$ low-pass), digitized (5000 Hz sampling rate, 16-bit resolution; M\&I), and displayed on screen and stored on hard drive. At the end of epicranial recordings, the skull was cleaned, the surgical wound sutured, and the mouse returned to its cage. During intracortical local field potential (LFP) recordings, a craniotomy was performed in the parietal bone, and a linear 16-electrode probe with $100 \mu \mathrm{m}$ interelectrode spacing (NeuroNexus Technologies) was inserted into the cortex perpendicular to its surface. Signals were amplified, filtered (1 Hz high-pass, $500 \mathrm{~Hz}$ low-pass), converted to digital (2000 $\mathrm{Hz}$ sampling rate, 16-bit resolution), and displayed online or stored on hard drive.

Rhythmic whisker stimulation. Baseline SEP were recorded three times every $10 \mathrm{~min}$ in mice previously housed in a poor or a rich environment. All whiskers on one side of the snout were then submitted during $10 \mathrm{~min}$ to rhythmic $1000 \mu \mathrm{m}$ back-and-forth deflections with $1 \mathrm{~ms}$ rise time. The rate of repetition of whisker deflection varied $(2,8$, or $20 \mathrm{~Hz})$. In controls, the stimulator was activated for $10 \mathrm{~min}$ close to the whiskers without making contact with them. Afterward, SEP were recorded as for the baseline measurements every $10 \mathrm{~min}$ for 60 to $90 \mathrm{~min}$.

Environmental conditions. Particular attention was brought to the housing conditions of our animals. Mice were kept in either a poor or a rich environment for 3 weeks before electrophysiological recordings. In the poor environment, a single mouse was housed alone in a standard cage $(30 \times 20 \mathrm{~cm})$. In the rich environment, a single male mouse was kept in one of five larger cages $(60 \times 30 \mathrm{~cm})$ containing two female conspecifics and several compartments and toys (with a different configuration in each cage). Male mice were moved to another enriched cage every 2-3 $\mathrm{d}$ to encourage exploration of a new environment and interaction with new partners. Except in the experiments concerned with the effects of environmental enrichment, all animals used in this study were housed in a poor environment before experiments were performed.

SEP waveform data processing and statistical analysis. The waveform showing the earliest latency and strongest response (located $1 \mathrm{~mm}$ pos- terior and $2.5 \mathrm{~mm}$ lateral to the bregma) was used for analysis. To decrease the intertrial variability of SEP waveforms and to reduce the number of stimuli needed to obtain stable recordings, a statistical outlier elimination procedure excluded those single trials where voltage values lay beyond 2 SDs of the mean for a given period, eliminating $~ 30 \%$ of trials (Sgro et al., 1990, Troncoso et al., 2000). The peak-to-peak amplitude between the initial largest positive and negative peaks occurring during the 10-30 ms poststimulus period of the SEP waveform was used for statistical analysis. Single comparisons between two groups or conditions were performed using $t$ tests, paired where appropriate, with Bonferroni correction to correct for test repetitions. Repeated measurements in the same animals were analyzed by repeated-measures ANOVA (Statistica 6; Statsoft). The significance level was set at $p=0.05$. Error bars represent SEM.

SEP mapping data processing and statistical analysis. Analysis was performed using the Cartool software (D. Brunet, Geneva University Hospital and Medical School, Geneva, Switzerland; http://brainmapping. unige.ch/Cartool.php). Before averaging, single trials were visually examined, and those that contained artifacts were excluded. Baseline correction was applied using the $50 \mathrm{~ms}$ prestimulus period as baseline. Averaged 31-channel SEP were recomputed against the average reference and analyzed as topographic maps of the surface electrical field generated by the brain. Surface voltage topography is a reference-free reflection of brain activity; differing map topographies are necessarily generated by differing patterns of neuronal activity (Lehmann, 1987). Graphical representations of surface voltage topography were created by interpolating values between electrodes using Delaunay triangle interpolation. To statistically evaluate map topography differences between conditions, a nonparametric randomization procedure was used (Murray et al., 2008). Briefly, under the null hypothesis, there is no topographic difference between maps from both conditions. Recorded maps from both conditions of each individual mouse at each time point were, therefore, first normalized with respect to the global amplitude of map voltage values, then randomly reallocated to either condition, and the spatial correlation between both conditions was quantified by computing the spatial dissimilarity (Murray et al., 2008). This procedure was repeated a large number of times to obtain a virtual distribution of the spatial dissimilarity under the null hypothesis. The spatial dissimilarity observed during the actual experiment was then compared with this distribution. The whole procedure was repeated for each time point between 0 and $40 \mathrm{~ms}$ after stimulus. The significance level was set at $p=0.05$ for at least 10 consecutive timeframes $(2 \mathrm{~ms})$. To localize the changes in map topography, in each condition amplitude-normalized maps of each mouse were averaged over time during periods of significant topographic difference, and paired randomization tests were then performed at each electrode between conditions. The significance level was set at $p=0.01$. Results are presented as topographic maps of the direction of significant differences between conditions. Because of the large number of test repetitions, results of point-wise statistical tests should be considered indicative of, rather than conclusive for, significant differences.

Intracortical current source density analysis and statistical analysis. Recordings from the 12 electrodes located between 0 and $1100 \mu \mathrm{m}$ depth were used for analysis. Before averaging, single trials were visually examined, and those that contained artifacts were excluded. Baseline correction was applied using the $50 \mathrm{~ms}$ prestimulus period as baseline. LFP recordings were further analyzed by computing the one-dimensional current source-density (CSD) perpendicular to cortical laminae. The CSD estimates local current flow between the intracellular and extracellular compartments, such as that generated by synaptic activity (Mitzdorf, 1985). It is calculated as the product of the second spatial derivative of voltage with the conductivity tensor. Since the latter is generally held to be constant in the neocortex, the CSD was here estimated by calculating the second spatial derivative using a 3-point finite-difference formula (Quairiaux et al., 2007). To compute the CSD at the extremities of the probe, virtual voltage values were extrapolated by assuming no voltage decay above the uppermost and below the lowermost electrodes (Vaknin et al., 1988; Heynen and Bear, 2001). Peaks of selected current sinks were used for statistical analysis. Repeated measurements in the same animals were analyzed by repeated-measures ANOVA (Statistica 6; 
A
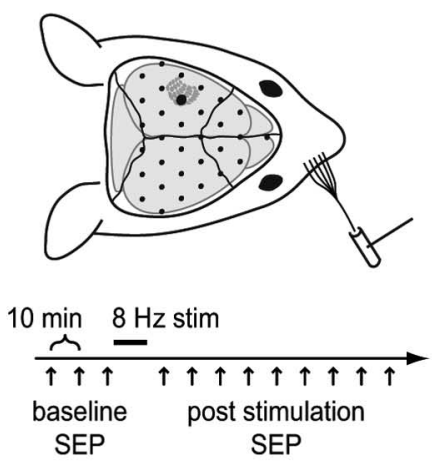

D

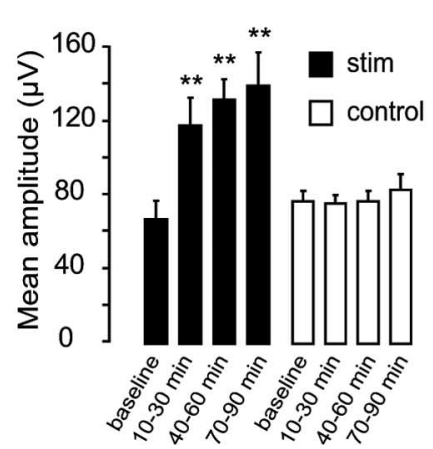

B

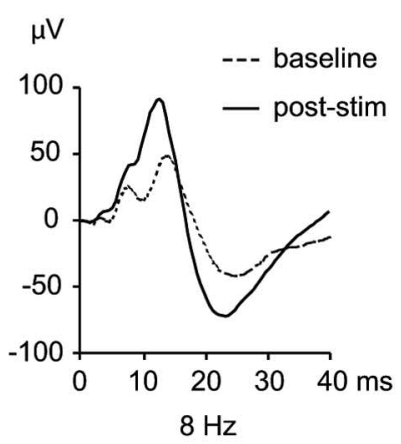

C

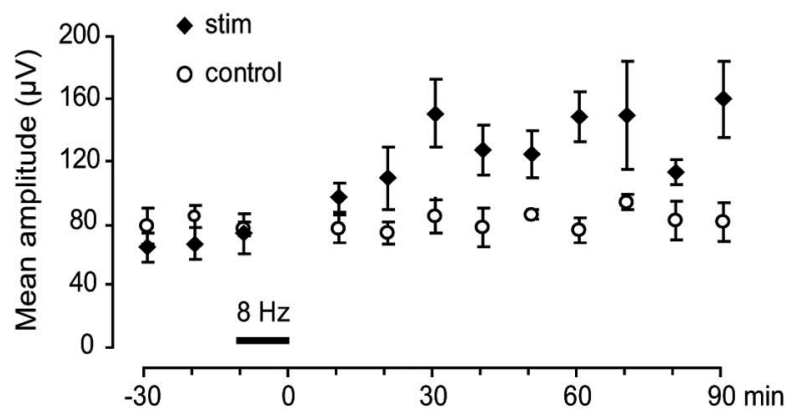

$\mathrm{E}$

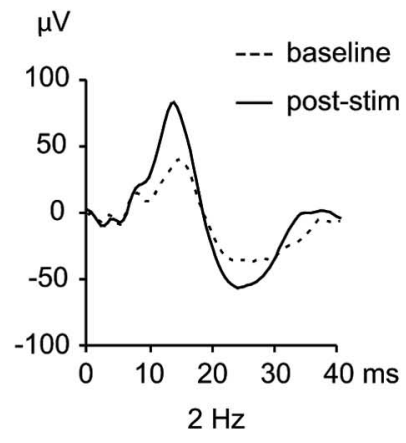

F $\quad \square$ baseline

$\mu \mathrm{V}$

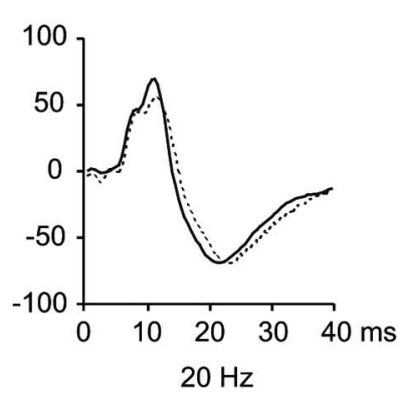

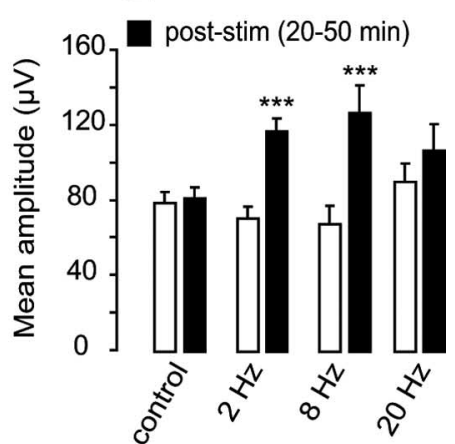

Figure 1. A 10 min period of $8 \mathrm{~Hz}$ rhythmic whisker stimulation induces a long-lasting plasticity of whisker-evoked brain responses. A, Top, Epicranial SEP recording setup. Anesthetized mice were placed in a stereotaxic frame; the scalp was retracted, and an array of 5-32 electrodes (black dots) was applied to the skull bones. These electrodes recorded potentials evoked by mechanical deflection of all large facial whiskers on the contralateral snout. The electrode recording the earliest-latency, largest-amplitude SEP (larger black dot) was used for analysis. Bottom, Rhythmic stimulation paradigm. Three baseline SEPs were recorded at $10 \mathrm{~min}$ intervals. The whiskers were then rhythmically deflected at a frequency of $8 \mathrm{~Hz}$ for $10 \mathrm{~min}$, after which SEP were again recorded every $10 \mathrm{~min}$ for $90 \mathrm{~min}$. In controls, the stimulator was activated for $10 \mathrm{~min}$ at $8 \mathrm{~Hz}$ close to the whiskers without making contact with them (sham stimulation). B, Examples of SEP waveforms recorded over the barrel cortex at baseline (dotted trace) and $30 \mathrm{~min}$ after a $10 \mathrm{~min}$ period of $8 \mathrm{~Hz}$ whisker stimulation (solid trace). C, Time course of SEP peak-to-peak averages before and after 8 $\mathrm{Hz}$ stimulation (stim; $n=5$ ) or sham stimulation (control; $n=5$ ). D, Pooled averages of the SEP peak-to-peak from the three baseline recordings and the recordings made between 10 and 30 , between 40 and 60 , and between 70 and $90 \mathrm{~min}$ after $8 \mathrm{~Hz}$ whisker stimulation. $\boldsymbol{E}$, Examples of SEP waveforms recorded over the barrel cortex at baseline and after rhythmic whisker stimulation at $2 \mathrm{~Hz}$ (left) or $20 \mathrm{~Hz}$ (right). $\boldsymbol{F}$, Pooled averages of the SEP amplitudes from the three baseline recordings and the recordings made between 20 and 50 min after the stimulation period. Significant difference from baseline at ${ }^{* *} p<0.01$ or at ${ }^{* * *} p<0.001$.

Statsoft). The significance level was set at $p=0.05$. Error bars represent SEM.

Histological control of electrode positioning. To verify the proper positioning of the intracortical probe, a lesion was made in the barrel or motor cortex at the end of the recordings by passing current $(20 \mu \mathrm{A}$ for $8 \mathrm{~s}$ ) through a glass microelectrode filled with Chicago Sky Blue dye and inserted at $400 \mu \mathrm{m}$ depth. Mice were then killed with pentobarbital, perfused with $4 \%$ paraformaldehyde, and the brains were extracted and processed for cytochrome oxidase histochemistry on tangential sections of the barrel cortex or for cresyl violet staining on coronal sections of the motor cortex using previously detailed protocols (Troncoso et al., 2004).

\section{Results}

Long-lasting, frequency-specific increase of somatosensory responses after $8 \mathrm{~Hz}$ whisker stimulation

We first explored the possibility that rhythmic whisker stimulation might lastingly influence the cortical processing of somatosensory input. For this purpose, we recorded the electrical response of the barrel cortex to mechanical deflection of contralateral whiskers (SEP) using minimally invasive epicranial electrodes applied to the surface of the skull bones under pentobarbital anesthesia (Fig. 1A) (Troncoso et al., 2000, 2004). The SEP mainly reflects synchronized postsynaptic events evoked by somatosensory stimuli in populations of cortical neurons; the successive voltage peaks of the SEP waveform (traditionally de- scribed by their amplitude and latency) are generated by partly distinct neuronal populations and reflect stages in the processing of sensory information (Allison et al., 1989a,b; Di and Barth, 1991; Peterson et al., 1995; Jellema et al., 2004).

To investigate whether rhythmic whisker stimulation influenced subsequent whisker-evoked responses, three baseline SEP were recorded, after which whiskers were rhythmically deflected at a frequency of $8 \mathrm{~Hz}$ for $10 \mathrm{~min}$. This rhythmic stimulation induced a twofold increase in SEP peak-to-peak amplitude over the next 90 min (Fig. $1 B, C$ ). In sham-stimulated control mice, in which the whisker stimulator was activated at $8 \mathrm{~Hz}$ for $10 \mathrm{~min}$ close to the whiskers without contacting them, no increase in SEP amplitude was evident over time (Fig. $1 C$ ) (test group, $p<0.001$; control group, $p>0.928$ for effect of recording time on SEP amplitude; repeated-measures ANOVA, $n=5$ in each group). This increase was visible already at the earliest poststimulus latencies ( $<10 \mathrm{~ms}$ poststimulus), where the SEP mostly reflects depolarization of layer IV cortical neurons by thalamocortical afferents from the ventroposteromedial (VPM) thalamic nucleus; it was even clearer between 10 and $30 \mathrm{~ms}$ poststimulus, where the SEP reflects disynaptic and plurisynaptic responses in supragranular and infragranular layers of the primary somatosensory cortex (Di et al., 1990; Jellema et al., 2004). SEP potentiation was 
A

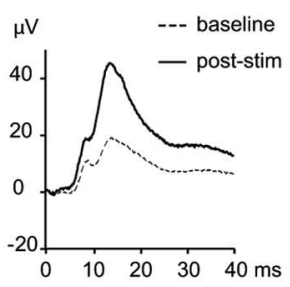

Left hemisphere
B

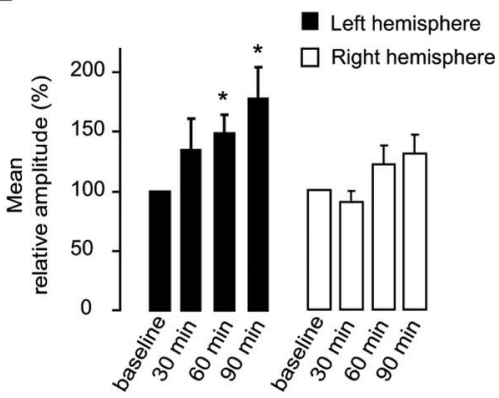

Figure 2. A $10 \mathrm{~min}$ period of $8 \mathrm{~Hz}$ rhythmic whisker stimulation induces a long-lasting and input-specific potentiation of whisker-evoked brain responses under isoflurane anesthesia. $\boldsymbol{A}$, Examples of SEP waveforms $(n=8)$ recorded under isoflurane anesthesia over the left and right barrel cortices in responses to respective contralateral deflections 20 min before (baseline) and 60 min after (post-stim) $8 \mathrm{~Hz}$ (right) whisker stimulation. B, Pooled averages of the SEP amplitudes from the three baseline and 30 , 60 , and $90 \mathrm{~min}$ after $8 \mathrm{~Hz}$ whisker stimulation, normalized against the baseline. Asterisks indicate significant differences with baseline at $p<0.05$. Potentiated responses are limited to the left barrel cortex, contralateral to the $8 \mathrm{~Hz}$ whisker stimulation.

already evident during the first $30 \mathrm{~min}$ after $8 \mathrm{~Hz}$ stimulation and was sustained for at least $90 \mathrm{~min}$ (Fig. 1D) (mean \pm SEM of SEP amplitude in tests: $67.83 \pm 9.96,118.40 \pm 15.51,132.85 \pm 15.51$, $140.41 \pm 18.0 \mu \mathrm{V}$ at baseline, $10-30,40-60$, and 70-90 min, respectively; $p=0.008, p=0.001, p=0.015$ for $10-30,40-60$, and 70-90 min against baseline, respectively; SEP amplitude in controls: $79.12 \pm 5.83,77.88 \pm 4.72,78.95 \pm 5.94,85.72 \pm 8.36$ $\mu \mathrm{V}$ at baseline, 10-30, 40-60, and 70-90 min, respectively; all $p>0.25$ for comparison against baseline; two-tailed paired $t$ tests with Bonferroni correction, $n=5$ in each group).

The effect of rhythmic whisker stimulation was more powerful for the $8 \mathrm{~Hz}$ frequency; $2 \mathrm{~Hz}$ stimulation induced a significant but smaller increase in SEP amplitude ( $8 \mathrm{~Hz}, 95 \%$ increase; $2 \mathrm{~Hz}$, $63 \%$ increase), whereas $20 \mathrm{~Hz}$ stimulation failed to induce any significant increase (Fig. $1 \mathrm{E}, \mathrm{F}$ ) (SEP amplitude for $2 \mathrm{~Hz}$ stimulation: $70.95 \pm 6.52,117.40 \pm 6.64 \mu \mathrm{V}$ at baseline and $20-50 \mathrm{~min}$ after stimulation, respectively, $p<0.001 ; 8 \mathrm{~Hz}$ stimulation: $67.83 \pm 9.96,127.43 \pm 14.63 \mu \mathrm{V}, p<0.001 ; 20 \mathrm{~Hz}$ stimulation: $90.35 \pm 9.81,107.34 \pm 13.88 \mu \mathrm{V}, p=0.07$; two-tailed paired $t$ tests with Bonferroni correction, $n=5$ in each group).

To demonstrate that $8 \mathrm{~Hz}$ whisker stimulation-induced potentiation of SEP responses was specific for the stimulated sensory input, we recorded SEP in response to deflection of whiskers on both sides of the snout before and after unilateral $8 \mathrm{~Hz}$ stimulation in a new group of eight animals. Since we used isoflurane anesthesia for the following intracortical and mapping analyses, we switched to isoflurane for this supplementary experimental group to ascertain that isoflurane did not suppress the effects of 8 $\mathrm{Hz}$ stimulation. Although SEP waveforms are known to differ depending on the particular anesthetic agent used (Rojas et al., 2006), $8 \mathrm{~Hz}$ stimulation under isoflurane anesthesia was followed by a long-lasting increase in the peak amplitude of responses to deflection of whiskers on the $8 \mathrm{~Hz}$-stimulated side (recorded in the hemisphere contralateral to $8 \mathrm{~Hz}$ stimulation) as observed under pentobarbital anesthesia (Fig. $2 A, B$ ) (increases of $34.3 \pm$ $26 \%, 48.5 \pm 16 \%$, and $78.6 \pm 26 \%$ at 30,60 , and 90 min compared with baseline, respectively, with $p=0.29, p=0.03$, and $p=$ 0.03 , respectively; two-tailed paired $t$ tests with Bonferroni correction). In contrast, responses to deflection of whiskers on the non- $8 \mathrm{~Hz}$-stimulated side (recorded in the hemisphere ipsilateral to $8 \mathrm{~Hz}$ stimulation) were not potentiated $(-9.6 \pm 9 \%,+21.3 \pm$ $16 \%$, and $29.6 \pm 23 \%$ at 30,60 , and 90 min compared with baseline, respectively, with $p=0.09, p=0.43$, and $p=0.23$, respectively), demonstrating that $8 \mathrm{~Hz}$ stimulation induced an input-specific potentiation of sensory responses rather than a generalized modulation of cortical responsiveness to sensory stimuli through attentional-like mechanisms. These results demonstrate that a short period of passive rhythmic somatosensory stimulation in the frequency range of exploratory whisking induces inputspecific, long-lasting plasticity of the cerebral processing of somatosensory input.

\section{Laminar profile of $8 \mathrm{~Hz}$}

stimulation-induced plasticity

To investigate how $8 \mathrm{~Hz}$ stimulation influences subsequent cortical somatosensory responses, we next turned to intracortical recordings. Inhaled isoflurane was preferred to maintain a more stable anesthesia over a longer time period. We then used multichannel intracortical probes to perform CSD analysis of the whisker-evoked LFP recorded in somatosensory cortical layers before and after $8 \mathrm{~Hz}$ stimulation. The CSD mainly reflects local synaptic activity by highlighting current flow in discrete cortical layers (Mitzdorf, 1985; Quairiaux et al., 2007). The baseline pattern of laminar whisker-evoked responses in the barrel cortex, starting at $\sim 6 \mathrm{~ms}$ after stimulus, was characterized by a current sink in layer IV that rapidly extended toward layers II-III, a fainter sink at the layer V-VI border, and superficial and deep sources (Fig. $3 A, B$, black CSD traces). This pattern is similar to previously reported findings in the rodent barrel cortex and reflects the propagation of excitatory synaptic activity in the barrel cortical column (Agmon and Connors, 1991; Armstrong-James et al., 1992; Jellema et al., 2004; Lecas, 2004). Stimulation (8 Hz) was followed by a significant increase in the amplitude of several sinks (Fig. 3A, red CSD traces). The largest increase was found in the superficial layer II-III sink (Fig. $3 C$, far left) $(p=0.006$ for effect of recording time on sink amplitude; repeated-measures ANOVA, $n=5$ ); smaller but significant increases were also observed in deep layer II-III (middle left; $p=0.011$ ), layer IV (middle right; $p=0.005$ ), and at the layer V-VI border (far right; $p=$ 0.005). No effect of recording time on sink amplitude was found in sham-stimulated controls (superficial layer II-III, $p=0.693$; deep layer II-III, $p=0.256$; layer IV, $p=0.819$; layer V-VI border, $p=0.700, n=5$ ). Superficial and deep sources, thought to represent the passive return current generated by excitatory synaptic activity, also increased in amplitude after $8 \mathrm{~Hz}$ stimulation (data not shown). These data indicate that long-term changes in whisker-evoked responses of the barrel cortex after 8 $\mathrm{Hz}$ stimulation mainly involved the granular and supragranular layers.

\section{Long-term plasticity after $8 \mathrm{~Hz}$ stimulation involves the sensorimotor network}

Cortical processing of somatosensory input is not limited to the primary somatosensory cortex, but involves several other cortical areas, such as the secondary somatosensory cortex, the vibrissal motor cortex, and somatosensory cortices of the opposite hemisphere, as well as subcortical structures (Kleinfeld et al., 2006; Ferezou et al., 2007). To investigate plasticity of large-scale sensorimotor networks, we mapped the surface potential field generated by brain activity using 32 epicranial electrodes distributed over the parietal and frontal bones of both hemispheres under isoflurane anesthesia (Mégevand et al., 2008). We then consid- 
A

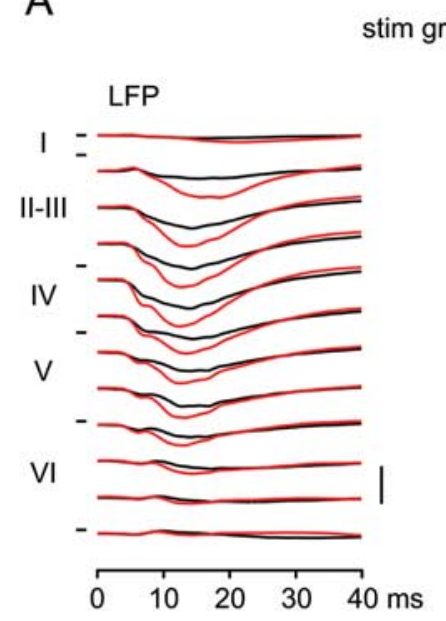

B

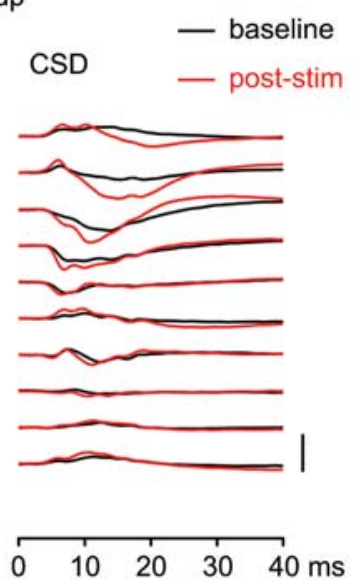

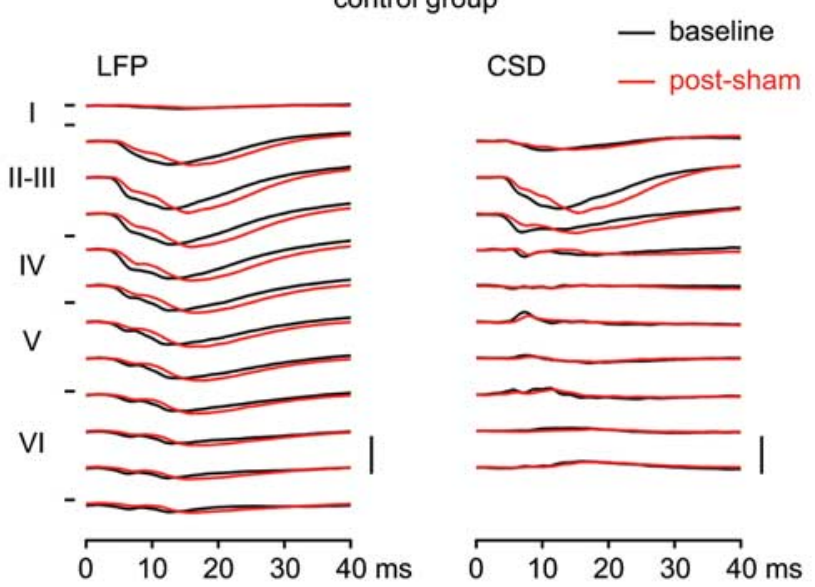

C superficial LII/III

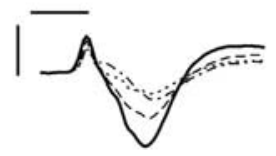
**

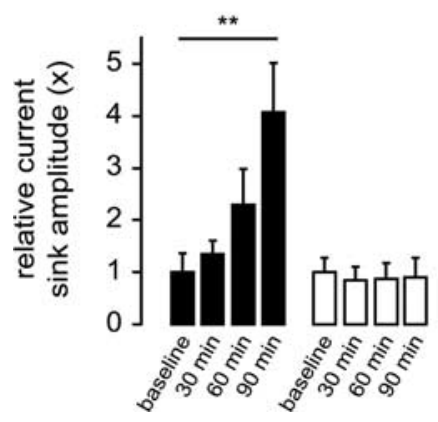

deep LII/III
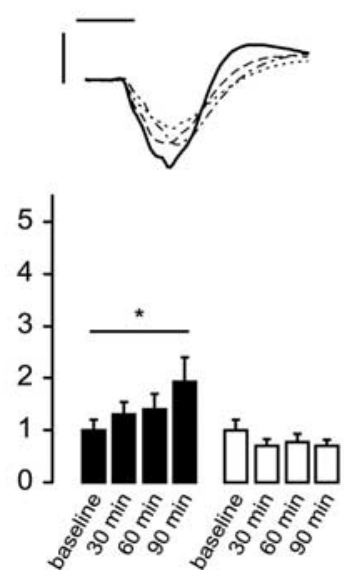

LIV

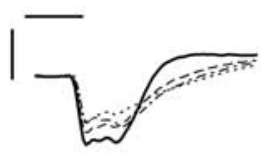

LV-VI border

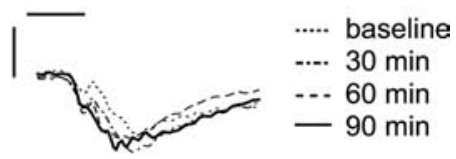

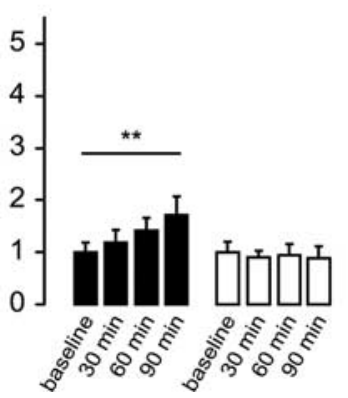

Figure 3. Rhythmic whisker stimulation induces long-lasting potentiation of whisker-evoked current sinks in the barrel cortex. $A$, Examples of whisker-evoked LFP recordings in the barrel cortex (left; $n=5$ mice) and CSD profiles (right) in the test group at baseline (black traces) and $60 \mathrm{~min}$ after $8 \mathrm{~Hz}$ whisker stimulation (red traces). Positive LFP voltages and CSD sources are upward going. Roman numerals indicate the approximate limits of cortical layers. Calibration: LFP, $250 \mu \mathrm{V} ; \mathrm{CSD}, 25 \mathrm{mV} / \mathrm{mm}^{2}$. B, Examples of whisker-evoked LFP recordings ( $\left.n=5 \mathrm{mice}\right)$ and CSD profiles in the control group at baseline and $60 \mathrm{~min}$ after sham whisker stimulation. Calibration as in A. C, Averages of selected current sink peak amplitudes at four selected electrodes recorded 30,60 , and 90 min after $8 \mathrm{~Hz}$ whisker stimulation, normalized against the baseline. Insets show the averaged CSD traces at the superficial layer II-III, deep layer II-III, layer IV, and layer V-VI border at baseline and 30, 60 , and $90 \mathrm{~min}$ after $8 \mathrm{~Hz}$ stimulation. Traces from controls are not shown. Calibration: horizontal, $10 \mathrm{~ms}$; vertical, $10 \mathrm{mV} / \mathrm{mm}^{2}$ for superficial and deep LII-III and LIV, $2.5 \mathrm{mV} / \mathrm{mm}{ }^{2}$ for LV-VI border. Significant difference from baseline at ${ }^{*} p<0.05$ or at ${ }^{* *} p<0.01$ (double).

ered the effect of $8 \mathrm{~Hz}$ stimulation on whisker-evoked responses in the sensorimotor network using topographic analysis of SEP maps. The topography of the surface potential map is a lowspatial resolution reflection of underlying cortical activity. The baseline whisker-evoked brain response started with activity in the parietal somatosensory cortex contralateral to stimulation (Fig. $4 A, B$, baseline maps at $7 \mathrm{~ms}$ after stimulus), followed by the appearance of activity in the frontal motor cortex (14.5 and $21 \mathrm{~ms}$ baseline maps) and also, faintly and briefly, in the somatosensory cortex of the hemisphere ipsilateral to stimulation (14.5 ms baseline maps). Later, activity was again mostly restricted to the somatosensory cortex contralateral to stimulation (30 ms baseline maps). A nonparametric randomization analysis of millisecondby-millisecond changes in map topography after $8 \mathrm{~Hz}$ stimulation indicated that SEP topography differed before and after stimulation from 9.5 to $12.5 \mathrm{~ms}$ and from 13.5 to $22.5 \mathrm{~ms}$ after stimulus (Fig. $4 C)(n=12)$. The randomization procedure is sensitive only to differences in map topography; maps with sim- ilar topography but different overall amplitude are not considered different. To localize the areas where whisker-evoked responses were affected by $8 \mathrm{~Hz}$ stimulation, a similar randomization procedure was then performed for voltage values at each electrode during the periods of significant difference in map topography. Whisker-evoked responses in the somatosensory cortex increased after $8 \mathrm{~Hz}$ stimulation (Fig. $4 E$ ), as seen in the waveform analysis of Figure 3, whereas responses in the frontal motor cortex decreased. No changes in SEP map topography were apparent in sham-stimulated controls (Fig. 4D) $(n=7)$. These results suggest that $8 \mathrm{~Hz}$ stimulation-induced plasticity was specific for contralateral sensorimotor networks.

Using intracortical recordings and CSD analysis to investigate the effect of $8 \mathrm{~Hz}$ stimulation on whisker-evoked responses in the motor cortex, we found that the baseline response to whisker stimulation consisted in a large current sink centered on deep supragranular layers, a fainter infragranular sink, and superficial and deep current sources (Fig. $5 A$, right), similar to previously 
A
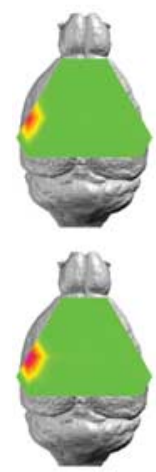

7
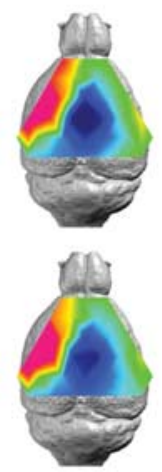

14.5 stim group
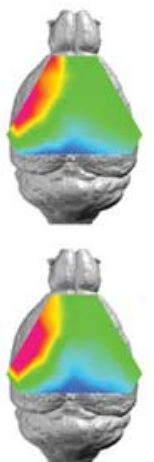

21
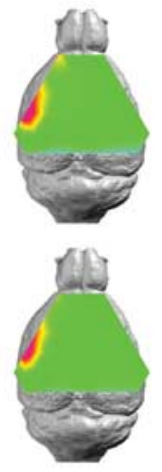

$30 \mathrm{~ms}$ baseline

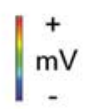

post-stim
C

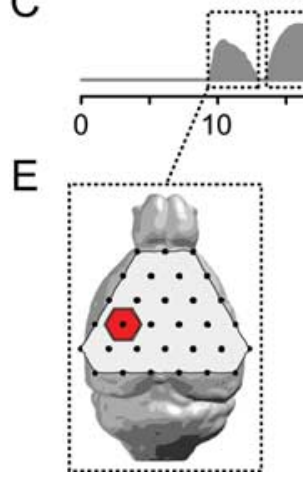

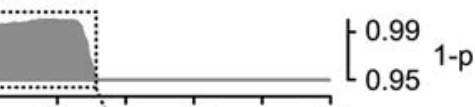

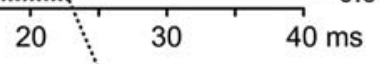

1-p

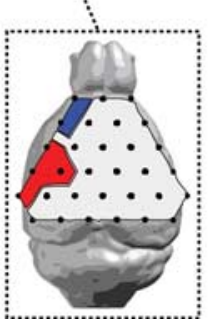

post-stim > baseline
0.05
$1 \quad p$
0.05
post-stim < baseline
B

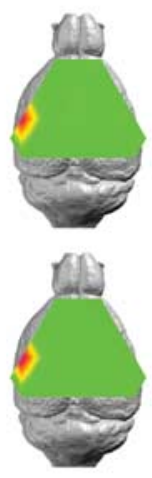

7 control group

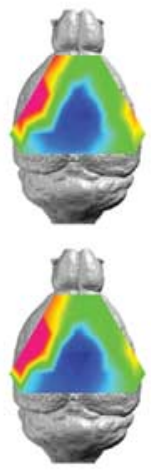

14.5
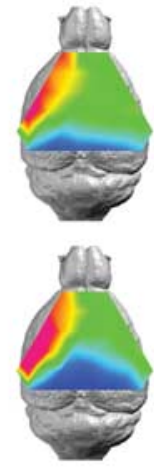

21

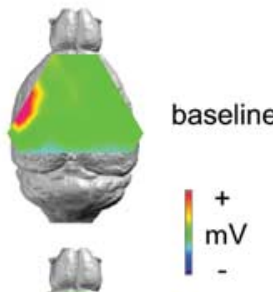

post-sham
D

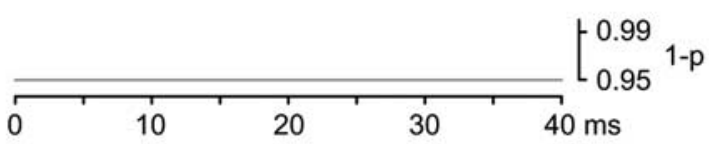

Figure 4. Rhythmic whisker stimulation induces a long-lasting plasticity of the topography of whisker-evoked multichannel brain responses. $A$, Topographic maps of the averaged SEP at baseline (top) and 40-60 min after rhythmic whisker stimulation (bottom). B, Topographic maps of the averaged SEP at baseline and 40-60 min after sham stimulation in controls. C, Results of the randomization procedure for topographic dissimilarity between SEP recorded at baseline and $40-60 \mathrm{~min}$ after $8 \mathrm{~Hz}$ whisker stimulation. 1-p values are plotted at each time point (5 time points per millisecond). $\boldsymbol{D}$, Results of the randomization procedure for topographic dissimilarity in sham-stimulated controls. E, Topographic maps (determined by an electrode-wise randomization procedure) of the directions of significant voltage differences at each electrode between the SEP at baseline and $40-60$ min after stimulation during the periods of significant differences in map topography. Red color indicates larger voltage values, whereas blue color indicates smaller voltage values after stimulation compared with baseline.

reported findings (Mégevand et al., 2008). In contrast to the increase observed in the somatosensory cortex, $8 \mathrm{~Hz}$ stimulation induced a significant decrease in the amplitude of the large supragranular sink in the motor cortex, whereas no decrease was observed in sham-stimulated controls (Fig. 5C) (stimulated group, $p=0.041$; control group, $p=0.608$ for effect of recording time on sink amplitude; repeated-measures ANOVA, $n=4$ in each group). These data indicate that $8 \mathrm{~Hz}$-induced plasticity in the motor cortex involved mainly the supragranular layers and was characterized by a decrease rather than an increase in response amplitude.

\section{Environmental enrichment occludes $8 \mathrm{~Hz}$ whisker} stimulation-induced plasticity of somatosensory responses Although rhythmic $8 \mathrm{~Hz}$ stimulation markedly influenced the subsequent processing of whisker-mediated input by the cortical somatosensory-motor network, the interpretation of the potential physiological role of this plasticity is complicated by the fact that whisker deflections were administered passively to anesthetized mice. To assess whether rhythmic sensory input-based plasticity might arise in more natural settings, we combined $8 \mathrm{~Hz}$ stimulation with environmental enrichment. We reasoned that an enriched environment would provide mice with more opportunities for active sensation, thereby increasing actively obtained whisker-mediated rhythmic somatosensory input, compared with the classic housing conditions obviously poor in terms of sensory stimulations. If plasticity were induced by this rhythmic input, it should interfere with the effects of subsequent passive 8 $\mathrm{Hz}$ whisker stimulation. Similar approaches have recently been used to establish a link between behavioral training or manipulation of sensory experience and neocortical long-term potentiation (Rioult-Pedotti et al., 2000; Clem et al., 2008).

We first characterized the effect of environmental enrichment on the SEP. A first group of mice were first housed alone in standard cages (poor environment) for 3 weeks, the same conditions as used for the first part of this study, after which the SEP was recorded. Taking advantage of the minimally invasive nature of epicranial SEP recordings (Troncoso et al., 2000), the same animals were then housed in larger cages containing several female conspecifics, compartments and toys for another 3 weeks (rich environment), and the SEP was recorded a second time. In a second group, mice were housed first in a rich and then in a poor environment (Fig. 6A). Two control groups consisted of mice kept in the same housing conditions for both 3 week periods. Shifting from a poor to a rich environment induced a significant, more than twofold increase in the SEP amplitude recorded over the barrel cortex (Fig. $6 B, D$ ) (SEP amplitude: $85.91 \pm 14.14$ vs $163.14 \pm 29.46 \mu \mathrm{V}, p=0.04$; two-tailed paired $t$ test, $n=5$ ) 
that was similar to that observed after $8 \mathrm{~Hz}$ stimulation in anesthetized mice (compare Fig. $6 B$ with Fig. $1 B$ ). In contrast, mice housed first in a rich and then in a poor environment showed a decrease in SEP amplitude from the first to the second recording (Fig. 6C,D) (SEP amplitude: $225.37 \pm 16.81$ vs $97.97 \pm 22.62 \mu \mathrm{V}, p<$ $0.001, n=5)$. Mice kept in the same housing conditions for both 3 week periods did not have any significant change in SEP amplitude (Fig. 6D) (“poor to poor": $84.92 \pm$ 14.14 vs $76.62 \pm 6.21 \mu \mathrm{V}, p=0.301$; "rich to rich": $169.1 \pm 21.35$ vs $184.89 \pm 29.87$ $\mu \mathrm{V}, p=0.153, n=4$ in each group). These results demonstrate that environmental enrichment reversibly influenced somatosensory-evoked responses.

We next investigated how environmental enrichment influenced $8 \mathrm{~Hz}$ whisker stimulation-induced plasticity. In mice housed for 3 weeks in a rich environment, $8 \mathrm{~Hz}$ whisker stimulation could not induce any further increase of SEP amplitude. In contrast, $8 \mathrm{~Hz}$ stimulation resulted in significantly enhanced SEP responses in mice housed in a poor environment (Fig. 6E,F) (rich environment: $163.84 \pm 19.26,193.6 \pm 31.69 \mu \mathrm{V}$ at baseline and $20-50 \mathrm{~min}$ after stimulation respectively, $p=0.305$; poor environment: $67.83 \pm 9.96,127.43 \pm 14.63 \mu \mathrm{V}$, $p=0.003$; two-tailed paired $t$ tests with Bonferroni correction, $n=5$ in each group). This suggests that the plasticity induced by environmental enrichment might share mechanistic features with that induced by $8 \mathrm{~Hz}$ whisker stimulation and raises the possibility that environmental enrichment saturated these mechanisms.

\section{Discussion}

The present study delineates experience-dependent plasticity events in large-scale somatosensory-motor networks after whisker stimulation in the adult mouse. We provide evidence that passive stimulation of whiskers at the naturally occurring $8 \mathrm{~Hz}$ frequency induced plasticity of subsequent whisker-evoked cortical responses. Changes after this rhythmic stimulation were long-lasting and frequency specific and involved widely distributed networks in the contralateral somatosensory and motor cortices. Intriguingly, changes in whisker-evoked potentials went in opposite directions in the somatosensory and motor cortices: although $8 \mathrm{~Hz}$ stimulation resulted in long-lasting potentiation in the barrel cortex, it induced depression in the motor cortex. Employing intracortical recordings and current source density analysis, we show that in both regions, the principal changes were localized to layers II/III and IV. Finally, environmental enrichment induced an increase of whisker-evoked responses in the somatosensory cortex that was similar to, and occluded the induction of further, potentiation elicited by passive rhythmic stimulation. Together, these results provide a tool for dissecting large-scale network properties that drive sensory stimulusdependent plasticity in the somatosensory-motor system. They indicate that rhythmically patterned natural stimuli can modify the cerebral processing of sensory information and suggest that this type of plasticity might play a role in learning during sensory perception.

In agreement with previous voltage-sensitive dye imaging data (Ferezou et al., 2007), our epicranial SEP mapping shows that whisker stimulation activated distributed neuronal networks in the hemispheres contralateral and ipsilateral to stimulation, both serially and in parallel. However, long-term plasticity induced by $8 \mathrm{~Hz}$ stimulation specifically involved contralateral circuits in the somatosensory barrel cortex and primary motor cortex, and potentiation occurred only in the somatosensory cortex. Intracortical recordings revealed that this increase was mainly related to an enlargement of local current flow in the granular and supragranular layers of the barrel cortex. These data are consistent with the notions that experience-dependent plasticity in the adult is predominantly cortical and that the layer IV to layer II/III pathway of the barrel cortex is an important locus of plasticity (Fox et al., 2002). The increased amplitude of the early latency current sink in layer IV of the barrel cortex also suggests that at least part of the potentiation in this system could be located at the thalamocortical synapse or at subcortical synapses. It should be noted here that, because SEP waveforms, particularly laterlatency components, are generated by the combined activity of several neuronal populations (Di et al., 1990; Jellema et al., 2004), waveform plasticity may reflect synaptic plasticity as well as plasticity occurring through other mechanisms, such as changes in neuronal excitability or in the activity of inhibitory interneurons 
A

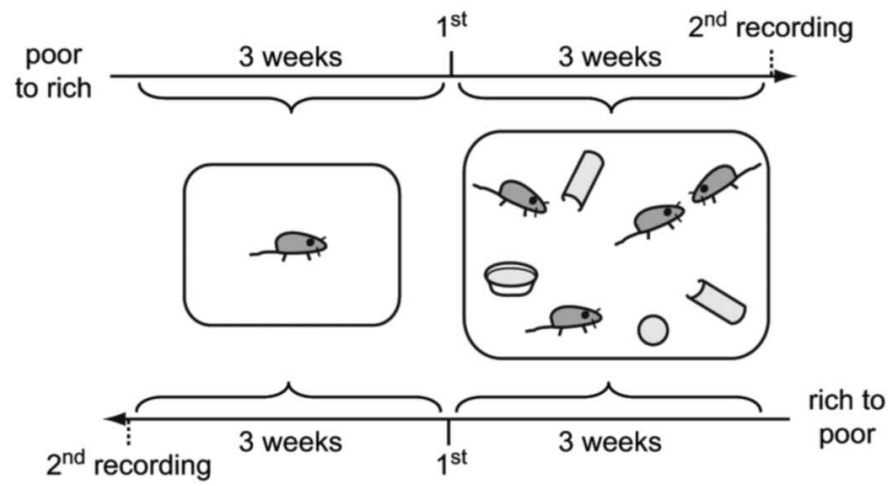

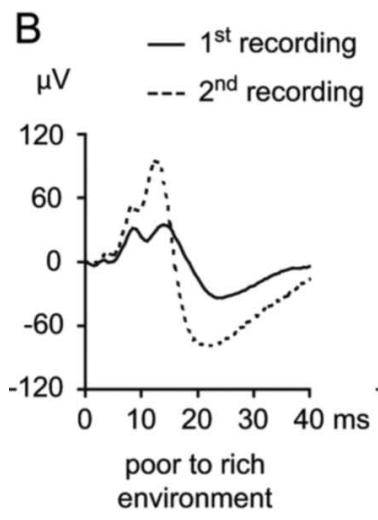

\section{C}

$$
\mu \mathrm{V}
$$

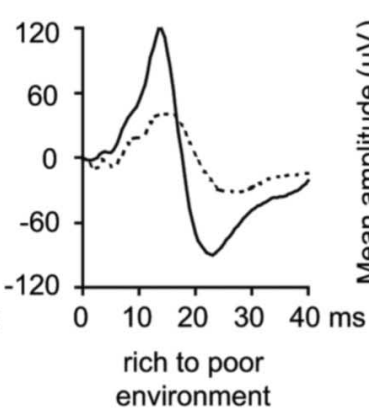

D
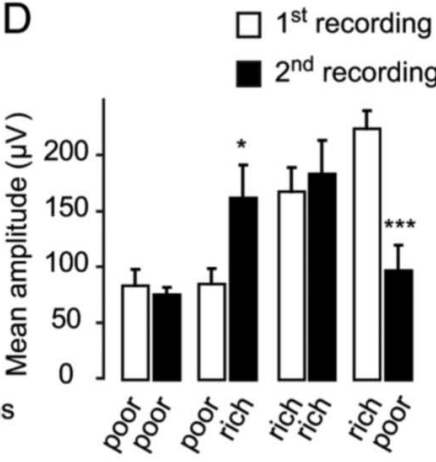

$\mathrm{F}$

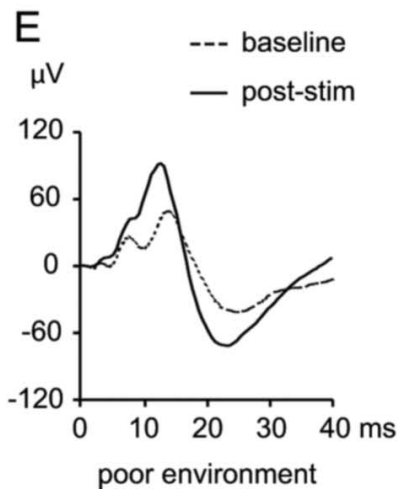

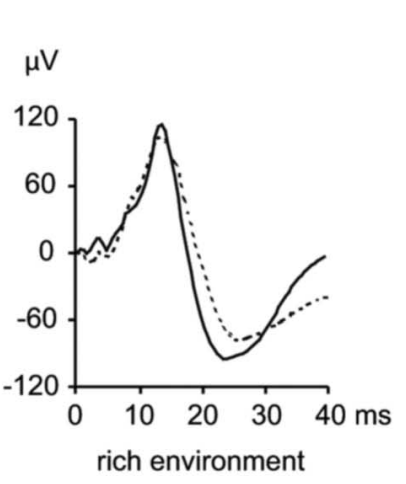

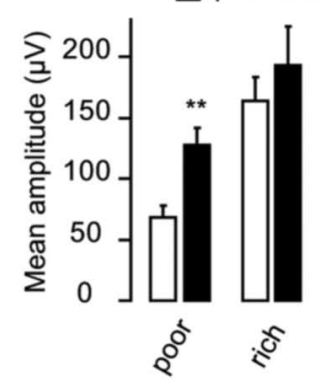

Figure 6. Changes in environmental conditions induce a reversible plasticity of whisker-evoked electrical brain responses. $\boldsymbol{A}$, Environmental enrichment paradigm. In one group, mice were housed alone in a standard cage for 3 weeks, after which the first SEP was recorded; mice were then housed in enriched cages for another 3 weeks, and the second SEP was recorded. In another group, mice were housed first in the rich and then in the poor environment. $\boldsymbol{B}$, Examples of SEP waveforms recorded over the barrel cortex in mice housed first in poor environment (solid trace) and then in a rich environment (dotted trace). $\boldsymbol{C}$, Examples of SEP waveforms in mice housed first in a rich environment (solid trace) and then in a poor environment (dotted trace). $\boldsymbol{D}$, Averages of the SEP amplitudes of the first and second recordings for the four experimental groups. $\boldsymbol{E}$, Examples of SEP waveforms recorded over the barrel cortex at baseline and after rhythmic $8 \mathrm{~Hz}$ whisker stimulation in mice previously housed in a poor environment (left; same dataset as in Fig. 1) or a rich environment (right). $\boldsymbol{F}$, Pooled averages of the SEP amplitudes from the three baseline recordings and the recordings made between 20 and $50 \mathrm{~min}$ after a $10 \mathrm{~min}$ period of $8 \mathrm{~Hz}$ whisker stimulation in mice previously housed in a poor or a rich environment.

(Clapp et al., 2006). Interestingly, earlier studies showed that rhythmically patterned bursts of electrical stimulation in thalamic sensory relay nuclei, with frequencies of $5-7 \mathrm{~Hz}$, could induce long-lasting potentiation of cortical responses to natural peripheral or single-pulse thalamic stimulation in the adult rat (Lee and Ebner, 1992; Heynen and Bear, 2001). The back-andforth whisker deflection we used during $8 \mathrm{~Hz}$ stimulation might cause several volleys of action potentials to be sent to the barrel cortex as a result of the rapid activation of several populations of direction-sensitive neurons in the trigeminal nerve (Lichtenstein et al., 1990), principal trigeminal nucleus (Minnery and Simons, 2003), and VPM thalamic nucleus (Simons and Carvell, 1989) and thereby induce cortical plasticity.

To our surprise, $8 \mathrm{~Hz}$ whisker stimulation also induced a long-lasting decrease in the amplitude of whisker-evoked responses in the supragranular layers of the motor cortex. The motor cortex is reciprocally connected with the barrel and secondary somatosensory cortices and with the posterior medial thalamic nucleus (Miyashita et al., 1994). The precise nature of sensory information transmitted through the barrel-to-motor cortex projection is unknown. However, the importance of this projection is illustrated by the observation that inactivating the barrel cortex widely suppresses somatosensoryevoked responses in the motor cortex (Chakrabarti et al., 2008). Our observation that whisker-evoked responses in layers II-III and (to a lesser extent) V of the barrel cortex, from which the projection to the motor cortex originates (Miyashita et al., 1994), were increased after $8 \mathrm{~Hz}$ stimulation suggests that the input from the barrel cortex to the motor cortex did not decrease but rather increased in amplitude. This raises the possibility that $8 \mathrm{~Hz}$ stimulation induced depression of subsequent whisker-evoked responses in the motor cortex. In brain slice experiments, the motor cortex is less susceptible to the induction of long-term potentiation than the somatosensory cortex (CastroAlamancos et al., 1995; Hess et al., 1996). This is in line with our observations and raises the possibility that the motor and barrel cortices adapt differentially to rhythmical input.

The molecular basis of $8 \mathrm{~Hz}$-induced plasticity remains to be elucidated. NMDA receptor activation-related mechanisms might be implicated, consistent with earlier findings that interfering with NMDA receptor function blocks cortical plasticity after rhythmic electrical thalamic stimulation (Heynen and Bear, 2001) or visual stimulation (Clapp et al., 2006). Acetylcholine signaling might also be important in modulating both the induction and the expression of rhythmic stimulation-related plasticity (Shulz et al., 2000). The momentary state of the cortex at the time of stimulus arrival is also likely to be crucial: whether a particular pattern of sensory input induces cortical plasticity was shown to depend on the phase of spontaneous cortical activity (Erchova and Diamond, 2004). The influence of rhythmic sensory input itself on ongoing cortical oscillations and the interactions between these phenomena with respect to plasticity remain to be elucidated.

An important new observation of the present study is that 
exposure of adult mice to an enriched environment resulted in a lasting increase in the amplitude of whisker-evoked responses in the somatosensory cortex and occluded further potentiation by 8 $\mathrm{Hz}$ stimulation. The effects of environmental enrichment on the SEP illustrates that interactions with the environment influence further sensory cerebral processing and are in line with previous findings of increased auditory-evoked potentials after auditory environmental enrichment in adult rats (Percaccio et al., 2007). Our results provide a strong argument in support of the idea that $8 \mathrm{~Hz}$-induced potentiation is behaviorally relevant. The occlusion of the effect of $8 \mathrm{~Hz}$ stimulation by environmental enrichment further indicates that rhythmic sensory inputs might play a role in shaping cortical processing in natural settings during active somatosensation. A similar occlusion of experimental plasticity by natural behavior is seen when testing long-term potentiation in brain slices prepared from rats previously trained on a sensorimotor task (Rioult-Pedotti et al., 2000). Likewise, the synaptic strengthening that occurs in the spared barrel column when all but one whisker are removed hinders subsequent generation of NMDA receptor-dependent long-term potentiation at layer IV to layer II-III synapses in mouse brain slices (Clem et al., 2008). Nevertheless, further plasticity remains possible at these synapses through metabotropic glutamate receptors (Clem et al., 2008), raising the possibility that several distinct plasticity mechanisms interact in shaping cortical processing as a function of sensory experience.

In conclusion, using surface potential mapping and intracortical recordings, we demonstrate the potential of naturally occurring rhythmic sensory stimuli to induce large-scale and longterm plasticity in cortical networks. To our knowledge, this is the first study that reports on the spatiotemporal dynamics of such stimulus-induced and frequency-specific plasticity in the mature somatosensory-motor cortex. Overall, our data, along with earlier reports in other sensory modalities and species (Clapp et al., 2005, 2006; Teyler et al., 2005), suggest that rhythmically patterned natural stimuli can change the cerebral processing of sensory information and that this type of plasticity might be a widespread feature of the mammalian brain. Future studies could focus on the physiological relevance of our findings. Specifically, it would be important to explore the implications of the plasticity that we identified at the large-scale network level with respect to synaptic plasticity, cortical receptor fields, perceptual learning and performance in sensorimotor tasks.

\section{References}

Agmon A, Connors BW (1991) Thalamocortical responses of mouse somatosensory (barrel) cortex in vitro. Neuroscience 41:365-379.

Allison T, McCarthy G, Wood CC, Darcey TM, Spencer DD, Williamson PD (1989a) Human cortical potentials evoked by stimulation of the median nerve. I. Cytoarchitectonic areas generating short-latency activity. J Neurophysiol 62:694-710.

Allison T, McCarthy G, Wood CC, Williamson PD, Spencer DD (1989b) Human cortical potentials evoked by stimulation of the median nerve. II. Cytoarchitectonic areas generating long-latency activity. J Neurophysiol 62:711-722.

Armstrong-James M, Fox K, Das-Gupta A (1992) Flow of excitation within rat barrel cortex on striking a single vibrissa. J Neurophysiol 68:1345-1358.

Brecht M, Schneider M, Sakmann B, Margrie TW (2004) Whisker movements evoked by stimulation of single pyramidal cells in rat motor cortex. Nature 427:704-710.

Carvell GE, Simons DJ (1990) Biometric analyses of vibrissal tactile discrimination in the rat. J Neurosci 10:2638-2648.

Castro-Alamancos MA, Donoghue JP, Connors BW (1995) Different forms of synaptic plasticity in somatosensory and motor areas of the neocortex. J Neurosci 15:5324-5333.
Chakrabarti S, Zhang M, Alloway KD (2008) MI neuronal responses to peripheral whisker stimulation: relationship to neuronal activity in SI barrels and septa. J Neurophysiol 100:50-63.

Clapp WC, Kirk IJ, Hamm JP, Shepherd D, Teyler TJ (2005) Induction of LTP in the human auditory cortex by sensory stimulation. Eur J Neurosci 22:1135-1140.

Clapp WC, Eckert MJ, Teyler TJ, Abraham WC (2006) Rapid visual stimulation induces N-methyl-D-aspartate receptor-dependent sensory longterm potentiation in the rat cortex. Neuroreport 17:511-515.

Clem RL, Celikel T, Barth AL (2008) Ongoing in vivo experience triggers synaptic metaplasticity in the neocortex. Science 319:101-104.

Crochet S, Petersen CC (2006) Correlating whisker behavior with membrane potential in barrel cortex of awake mice. Nat Neurosci 9:608-610.

Di S, Barth DS (1991) Topographic analysis of field potentials in rat vibrissa/ barrel cortex. Brain Res 546:106-112.

Di S, Baumgartner C, Barth DS (1990) Laminar analysis of extracellular field potentials in rat vibrissa/barrel cortex. J Neurophysiol 63:832-840.

Erchova IA, Diamond ME (2004) Rapid fluctuations in rat barrel cortex plasticity. J Neurosci 24:5931-5941.

Fee MS, Mitra PP, Kleinfeld D (1997) Central versus peripheral determinants of patterned spike activity in rat vibrissa cortex during whisking. J Neurophysiol 78:1144-1149.

Ferezou I, Haiss F, Gentet LJ, Aronoff R, Weber B, Petersen CC (2007) Spatiotemporal dynamics of cortical sensorimotor integration in behaving mice. Neuron 56:907-923.

Fox K, Wallace H, Glazewski S (2002) Is there a thalamic component to experience-dependent cortical plasticity? Philos Trans R Soc Lond B Biol Sci 357:1709-1715.

Fries P (2005) A mechanism for cognitive dynamics: neuronal communication through neuronal coherence. Trends Cogn Sci 9:474-480.

Gilbert CD, Sigman M, Crist RE (2001) The neural basis of perceptual learning. Neuron 31:681-697.

Hess G, Aizenman CD, Donoghue JP (1996) Conditions for the induction of long-term potentiation in layer II/III horizontal connections of the rat motor cortex. J Neurophysiol 75:1765-1778.

Heynen AJ, Bear MF (2001) Long-term potentiation of thalamocortical transmission in the adult visual cortex in vivo. J Neurosci 21:9801-9813.

Jellema T, Brunia CH, Wadman WJ (2004) Sequential activation of microcircuits underlying somatosensory-evoked potentials in rat neocortex. Neuroscience 129:283-295.

Kleinfeld D, Sachdev RN, Merchant LM, Jarvis MR, Ebner FF (2002) Adaptive filtering of vibrissa input in motor cortex of rat. Neuron 34:1021-1034.

Kleinfeld D, Ahissar E, Diamond ME (2006) Active sensation: insights from the rodent vibrissa sensorimotor system. Curr Opin Neurobiol $16: 435-444$.

Lecas JC (2004) Locus coeruleus activation shortens synaptic drive while decreasing spike latency and jitter in sensorimotor cortex. Implications for neuronal integration. Eur J Neurosci 19:2519-2530.

Lee SM, Ebner FF (1992) Induction of high frequency activity in the somatosensory thalamus of rats in vivo results in long-term potentiation of responses in SI cortex. Exp Brain Res 90:253-261.

Lehmann D (1987) Principles of spatial analysis. In: Handbook of electroencephalography and clinical neurophysiology, Vol 1. Methods of analysis of brain electrical and magnetic signals (Gevins AS, Rémond A, eds), pp 309-354. Amsterdam: Elsevier.

Lichtenstein SH, Carvell GE, Simons DJ (1990) Responses of rat trigeminal ganglion neurons to movements of vibrissae in different directions. Somatosens Mot Res 7:47-65.

Mégevand P, Quairiaux C, Lascano AM, Kiss JZ, Michel CM (2008) A mouse model for studying large-scale neuronal networks using EEG mapping techniques. Neuroimage 42:591-602.

Minnery BS, Simons DJ (2003) Response properties of whisker-associated trigeminothalamic neurons in rat nucleus principalis. J Neurophysiol $89: 40-56$

Mitzdorf U (1985) Current source-density method and application in cat cerebral cortex: investigation of evoked potentials and EEG phenomena. Physiol Rev 65:37-100.

Miyashita E, Keller A, Asanuma H (1994) Input-output organization of the rat vibrissal motor cortex. Exp Brain Res 99:223-232.

Murray MM, Brunet D, Michel CM (2008) Topographic ERP analyses: a step-by-step tutorial review. Brain Topogr 20:249-264. 
Percaccio CR, Pruette AL, Mistry ST, Chen YH, Kilgard MP (2007) Sensory experience determines enrichment-induced plasticity in rat auditory cortex. Brain Res 1174:76-91.

Peterson NN, Schroeder CE, Arezzo JC (1995) Neural generators of early cortical somatosensory evoked potentials in the awake monkey. Electroencephalogr Clin Neurophysiol 96:248-260.

Quairiaux C, Armstrong-James M, Welker E (2007) Modified sensory processing in the barrel cortex of the adult mouse after chronic whisker stimulation. J Neurophysiol 97:2130-2147.

Rioult-Pedotti MS, Friedman D, Donoghue JP (2000) Learning-induced LTP in neocortex. Science 290:533-536.

Rojas MJ, Navas JA, Rector DM (2006) Evoked response potential markers for anesthetic and behavioral states. Am J Physiol Regul Integr Comp Physiol 291:R189-R196.

Sgro JA, Emerson RG, Stanton PC (1990) Advanced techniques of evoked potential acquisition and processing. In: Evoked potentials in clinical medicine, Ed 2 (Chiappa KH, ed). New York: Raven.

Shulz DE, Sosnik R, Ego V, Haidarliu S, Ahissar E (2000) A neuronal analogue of state-dependent learning. Nature 403:549-553.

Simons DJ, Carvell GE (1989) Thalamocortical response transformation in the rat vibrissa/barrel system. J Neurophysiol 61:311-330.

Teyler TJ, Hamm JP, Clapp WC, Johnson BW, Corballis MC, Kirk IJ (2005)
Long-term potentiation of human visual evoked responses. Eur J Neurosci 21:2045-2050.

Troncoso E, Muller D, Czellar S, Zoltan Kiss J (2000) Epicranial sensory evoked potential recordings for repeated assessment of cortical functions in mice. J Neurosci Methods 97:51-58.

Troncoso E, Muller D, Korodi K, Steimer T, Welker E, Kiss JZ (2004) Recovery of evoked potentials, metabolic activity and behavior in a mouse model of somatosensory cortex lesion: role of the neural cell adhesion molecule (NCAM). Cereb Cortex 14:332-341.

Vaknin G, DiScenna PG, Teyler TJ (1988) A method for calculating current source density (CSD) analysis without resorting to recording sites outside the sampling volume. J Neurosci Methods 24:131-135.

Varela F, Lachaux JP, Rodriguez E, Martinerie J (2001) The brainweb: phase synchronization and large-scale integration. Nat Rev Neurosci 2:229-239.

von Heimendahl M, Itskov PM, Arabzadeh E, Diamond ME (2007) Neuronal activity in rat barrel cortex underlying texture discrimination. PLoS Biol 5:e305.

Wolfe J, Hill DN, Pahlavan S, Drew PJ, Kleinfeld D, Feldman DE (2008) Texture coding in the rat whisker system: slip-stick versus differential resonance. PLoS Biol 6:e215. 